\title{
Synthesis of the Organic/Inorganic Hybrid Star Polymers and Their Inclusion Complexes with Cyclodextrins
}

\author{
Shih-Chi Chan, Shiao-Wei Kuo, and Feng-Chih Chang* \\ Institute of Applied Chemistry, National Chiao TungUniversity, Hsin Chu, Taiwan \\ Received January 7, 2005; Revised Manuscript Received February 17, 2005
}

\begin{abstract}
In this study, we synthesized a series of the organic/inorganic hybrid star PCLs. These star PCLs can form inclusion complexes (ICs) with $\alpha$ - and $\gamma$-CD, but not with $\beta$-CD. These CD ICs were characterized by XRD, solid-state ${ }^{13} \mathrm{C}$ CP/MAS NMR spectroscopy, ${ }^{1} \mathrm{H}$ NMR spectroscopy, FT-IR spectroscopy, DSC, and TGA. Our results suggest that the PCL chains of these star polymers lose their original crystalline properties and were included inside the channels provided by the CDs to form a columnar crystalline structures. The stoichiometries (PCL:CD) that we determined by ${ }^{1} \mathrm{H}$ NMR spectroscopy for all of the ICs with $\alpha$ - or $\gamma$-CD are higher than those of the corresponding CD/linear PCL ICs because of the steric hindrance around the bulky POSS core, which causes some of the $\epsilon$-caprolactone units near the core to be free from complexation with the CDs. From these analyses, we proposed some possible structures for the CD/star PCL ICs.
\end{abstract}

\section{Introduction}

In recent years, supramolecular polymer chemistry has emerged as a fascinating new field of macromolecular research. A number of unique supramolecular architectures, such as molecular tubes or polyrotaxanes based on inclusion complexes (ICs) of polymer chains threaded through cyclic components, have been prepared as novel composite host/guest polymeric assemblies. $^{1-4}$ Cyclodextrin (CD) is one of the most popular host molecules employed to construct molecular assemblies. CDs are cyclic oligosaccharides consisting of six $(\alpha)$, seven $(\beta)$, or eight $(\gamma)$ glucose units linked by 1,4- $\alpha$-glucosidic bonds. ${ }^{5}$ They have rigid, well-defined ring structures and the ability to bind various compounds. The cone-shaped cavities of CDs can act as hosts for a great variety of molecular guests. ${ }^{6}$ The driving forces for polyrotaxane formation are believed to be intermolecular hydrogen bonding between neighboring CDs as well as geometric compatibility and hydrophobic interactions between CDs and polymers. ${ }^{7-9}$

Supramolecular inclusion complexes stabilized by noncovalent interactions can be formed by threading CD molecules onto polymer chains. The first inclusion complex of a CD and a polymer, reported ${ }^{10}$ by Harada et al. in 1990, was that of $\alpha-C D$ forming a crystalline stoichiometric IC with poly(ethylene glycol). Since this initial discovery of $\mathrm{CD} /$ polymer ICs, several reports have described how many linear polymeric guest, possessing either hydrophilic or hydrophobic properties, have the ability to form ICs with the different types of CDs. ${ }^{1-32,41}$ Although the depths of the cavities for the three CD molecules are the same $(7.9 \AA)$, their cavity diameters are different: ca. 4.5, 7, and 8.5-9.0 $\AA$ for $\alpha-, \beta$-, and $\gamma$-CD, respectively. ${ }^{9}$ Harada et al. demonstrated that a good correlation exists between the cross-sectional areas of the polymers and the cavity sizes of the CDs. ${ }^{18}$ For example, poly(ethylene glycol) (PEG) forms inclusion complexes with $\alpha-\mathrm{CD}^{10,19}$ and $\gamma-\mathrm{CD},{ }^{9}$ whereas poly(propylene glycol) (PPG) forms inclusion complexes only with $\beta$-CD in most conditions. ${ }^{20,21} \mathrm{CDs}$ also form crys-

* To whom corresponding should be addressed: Tel 886-35131512; Fax 886-3-5719507; e-mail changfc@mail.nctu.edu.tw. talline complexes with other polymers, including poly(methyl vinyl ether), ${ }^{22}$ polyisobutylene, ${ }^{23}$ poly(trimethylene adipate), ${ }^{24}$ poly(hydroxybutyrate), ${ }^{25}$ poly( $\epsilon$-carolactone), ${ }^{26}$ and nylon- $6 .{ }^{27}$ Because of the long chainlike nature of polymers, all of the crystalline structures of polymer/CD ICs are expected to exist in columnar forms. ${ }^{28}$ As Tonelli et al. indicated, the study of these types of inclusion complexes provides an oppotunity to investigate the behavior of single polymer chains or two adjacent parallel chains in isolated and well-defined environments. ${ }^{29,30}$

Recently, reports have described columnar-structured ICs of CDs combined with organic-inorganic materials, such as PDMS ${ }^{31}$ and PSis. ${ }^{32}$ Organic/inorganic hybrid nanocomposite materials have drawn a great deal of attention because of the length scales involved and the observed enhancement of material properties relative to those of the corresponding nonhybird materials. ${ }^{33,34}$ Nanostructured polyhedral oligomeric silsesquioxanes (POSS) and $\mathrm{Q}_{8} \mathrm{M}_{8}{ }^{\mathrm{H}}$ species have been used to prepare designed novel nanocomposites that have a variety of potential applications. ${ }^{33-36}$ The nanocages of the silsesquioxanes consist of a rigid, crystalline, and welldefined inorganic core, to which as many as eight identical covalently bounded polymerizable functional groups can be appended. ${ }^{37,38}$ The sizes of the cubic core $(0.53 \mathrm{~nm})$ and the functionalized arms $(1-2 \mathrm{~nm}$, depending on the structure) define the dimensions of the organic/inorganic components in the resulting nanocomposites in which both components are distributed uniformly and periodically. ${ }^{39}$

Poly( $\epsilon$-caprolactone) (PCL) is one of the most attractive and promising synthetic polymers becaused it is biodegradable when in contact with microorganisms, and thus, it has potential importance in a number of different applications. ${ }^{40}$ PCL forms columnar-structured inclusion complexes with both $\alpha-\mathrm{CD}$ and $\gamma-\mathrm{CD}$ in high yield; in the $\gamma$-CD/PCL complex, two adjacent PCL chains are threaded within each $\gamma$-CD channel. ${ }^{41}$ In this study, we combined chains of PCL with inorganic POSS to develop organic-inorganic hybrid star macromolecules through living ring-opening polymerization. We were interested in investigating whether such 
organic/inorganic star polymers can also form crystalline inclusion complexes with CDs. This paper describes the preparation and characterization of inclusion complexes of CDs with the synthesized organic/inorganic hybrid star PCLs; the mode of the inclusion is discussed in detail.

\section{Experimental Section}

Materials. $\mathrm{Q}_{8} \mathrm{M}_{8}{ }^{\mathrm{H}} \quad\{1,3,5,7,9,11,13,15$-octakis (dimethylsilyloxy)pentacyclo[9.5.1.1.3.9. $\left.1^{5.15} \cdot 1^{7.13}\right]$ octasiloxane $\}$ was purchased from Hybrid Plastics. Allyl alcohol, imidazole, tertbutyldimethylsilyl chloride, tetrabutylammonium fluoride, platinum divinyltetramethylsidiloxane [Pt(dvs)], and tin(II) 2-ethyl-hexanoate [Sn(oct $\left.)_{2}\right]$ were obtained from Aldrich. Acetic acid, DMF, toluene, and THF were purchased from TEDIA Co., and $\epsilon$-caprolactone was obtained form Acros Organics. $\alpha$-CD and $\gamma$-CD were obtained from Tokyo Kasei, Tokyo. All CDs were dried at $80{ }^{\circ} \mathrm{C}$ in vacuo for 2 days prior to use. Other chemicals were used as received without further purification.

Silylation: Synthesis of 3-(tert-Butyldimethylsilyloxy)propene. ${ }^{42}$ Imidazole $(23.14 \mathrm{~g}, 0.34 \mathrm{~mol})$ was added to a solution of allyl alcohol (9.52 mL, $0.14 \mathrm{~mol})$ and tert-butyldimethylsilyl chloride $(26.11 \mathrm{~g}, 0.173 \mathrm{~mol})$ in dry DMF $(100 \mathrm{~mL})$. The mixture was stirred overnight at ambient temperature under nitrogen and then poured into ice-water to stop the reaction. The organic layer was extracted with diethyl ether, washed with distilled water, $5 \%$ aqueous $\mathrm{NaHCO}_{3}$, and distilled water, and then dried $\left(\mathrm{MgSO}_{4}\right)$. Evaporation of the residual material and solvent gave a viscous solution that was dried under vacuum for 3 days to give the product $(22.3 \mathrm{~g}$, 92\%). Selected characterization data: ${ }^{1} \mathrm{H}$ NMR $(500 \mathrm{MHz}$, $\left.\mathrm{CDCl}_{3}, \mathrm{CHCl}_{3} \mathrm{ref}\right): \delta 5.89\left(\mathrm{~m}, 1 \mathrm{H}, \mathrm{CH}_{2} \mathrm{CH}\right), 5.24\left(\mathrm{q}, 1 \mathrm{H}, \mathrm{CH}^{1} \mathrm{H}^{2-}\right.$ $\mathrm{CH}), 5.03\left(\mathrm{q}, 1 \mathrm{H}, \mathrm{CH}^{1} \mathrm{H}^{2} \mathrm{CH}\right), 0.85\left[\mathrm{~m}, 9 \mathrm{H}, \mathrm{SiC}\left(\mathrm{CH}_{3}\right)_{4}\right], 0.046$ $\left[\mathrm{m}, 6 \mathrm{H}, \mathrm{Si}\left(\mathrm{CH}_{3}\right)_{2}\right]$. FTIR $\left(\mathrm{KBr}, \mathrm{cm}^{-1}\right) v_{\mathrm{C}-\mathrm{H}}: 2959 \mathrm{~s}, 2931 \mathrm{~s}, 2875$ $\mathrm{m} ; v_{\mathrm{C}=\mathrm{C}}: 1410 \mathrm{~s}, 980 \mathrm{~s}, 940 \mathrm{~s} ; v_{\mathrm{CH}_{3}}: 1258 \mathrm{~m} ; v_{\mathrm{Si}-\mathrm{C}}: 1173 \mathrm{~m}$; $\nu_{\mathrm{Si}-\mathrm{O}}: 1082 \mathrm{~s} ; v_{\mathrm{C}-\mathrm{O}-\mathrm{Si}}: 1010 \mathrm{~s}$ (presented in Figures 1a and 2a).

Synthesis of Octakis(tert-butyldimethylsiloxylpropyldimethylsiloxy)octasilsesquioxane. ${ }^{43} \mathrm{Q}_{8} \mathrm{M}_{8}{ }^{\mathrm{H}}(5.00 \mathrm{~g}, 4.91$ mmol) was placed in a Schlenk flask equipped with a reflux condenser and a magnetic stirrer. Toluene $(25 \mathrm{~mL})$ was added to dissolve the sample, and then 3-(tert-butyldimethylsilyloxy)propene ( $8.47 \mathrm{~g}, 49.1 \mathrm{mmol}$ ) was added. $\mathrm{Pt}(\mathrm{dvs})$ (5 drops) was added via a syringe. The mixture was then quickly brought to reflux under nitrogen and then maintained at $100{ }^{\circ} \mathrm{C}$ for $4 \mathrm{~h}$. Activated carbon was added to deactivate the $\mathrm{Pt}(\mathrm{dvs})$ catalyst, and then the mixture was stirred for $1 \mathrm{~h}$. Residual allyl alcohol and toluene were removed by rotary evaporation and then dried under vacuum for 3 days to provide octakis(tert-butyldimethylsiloxylpropyldimethylsiloxy)octasilsesquioxane (10.3 g, 88\%). Selected characterization data: ${ }^{1} \mathrm{H}$ NMR $(500 \mathrm{MHz}$, $\left.\mathrm{CDCl}_{3}, \mathrm{CHCl}_{3} \mathrm{ref}\right): \delta 3.53\left(\mathrm{t}, 16 \mathrm{H}, \mathrm{CH}_{2} \mathrm{CH}_{2} \mathrm{O}\right), 1.53(\mathrm{t}, 16 \mathrm{H}$, $\left.\mathrm{CH}_{2} \mathrm{CH}_{2} \mathrm{O}\right), 0.84\left[\mathrm{~m}, 72 \mathrm{H}, \mathrm{SiC}\left(\mathrm{CH}_{3}\right)_{4}\right], 0.54\left(\mathrm{~m}, 16 \mathrm{H}, \mathrm{SiCH}_{2-}\right.$ $\left.\mathrm{CH}_{2}\right), 0.048\left[\mathrm{~m}, 96 \mathrm{H}, \mathrm{Si}\left(\mathrm{CH}_{3}\right)_{2}\right]$. FTIR $\left(\mathrm{KBr}, \mathrm{cm}^{-1}\right) v_{\mathrm{C}-\mathrm{H}}: 2959$ $\mathrm{s}, 2931 \mathrm{~s}, 2875 \mathrm{~m} ; v_{\mathrm{CH}_{3}}: 1258 \mathrm{~m} ; v_{\mathrm{Si}-\mathrm{C}}: 1173 \mathrm{~m} ; v_{\mathrm{Si}-\mathrm{O}}: 1082 \mathrm{~s}$; $v_{\mathrm{C}-\mathrm{O}-\mathrm{Si}}: 1010 \mathrm{~s}$ (displayed in Figures $1 \mathrm{c}$ and $2 \mathrm{c}$; the ${ }^{1} \mathrm{H}$ NMR and FTIR spectra of the pure $\mathrm{Q}_{8} \mathrm{M}_{8} \mathrm{H}$ are presented in Figures $1 \mathrm{~b}$ and $2 \mathrm{~b}$ ).

Desilylation: Synthesis of Octakis(3-hydroxypropyldimethylsiloxy)octasilsesquioxane (OHPS). Octakis(tertbutyldimethylsiloxylpropyldimethylsiloxy)octasilsesquioxane (3 g) was dissolved in a mixture of THF and acetic acid (10:1) and then tetrabutylammonium fluoride $(2 \mathrm{~mol}$ per tert-butyldimethylsiloxyl group). The mixture was stirred under nitrogen at ambient temperature for $2 \mathrm{~h}$. Following evaporation of the solvent, the viscous product was precipitated into water with vigorous stirring, collected by vacuum filtration, and repeatedly washed with water. The product was further dried under vacuum for 3 days to provide OHPS as a white powder (1.51 g, 81\%). Selected characterization data: ${ }^{1} \mathrm{H}$ NMR (500 $\mathrm{MHz}, \mathrm{CDCl}_{3}, \mathrm{CHCl}_{3}$ ref): $\delta 3.58$ (q, $16 \mathrm{H}, \mathrm{CH}_{2} \mathrm{CH}_{2} \mathrm{OH}$ ), $1.62\left(\mathrm{~m}, 16 \mathrm{H}, \mathrm{CH}_{2} \mathrm{CH}_{2} \mathrm{CH}_{2}\right), 0.69\left(\mathrm{~m}, 16 \mathrm{H}, \mathrm{CH}_{2} \mathrm{CH}_{2} \mathrm{CH}_{2}\right), 0.15$ [s, $\left.48 \mathrm{H}, \mathrm{Si}\left(\mathrm{CH}_{3}\right)_{2}\right]$. FTIR $\left(\mathrm{KBr}, \mathrm{cm}^{-1}\right) v_{\mathrm{OH}}: 3318 \mathrm{~s} ; v_{\mathrm{C}-\mathrm{H}}: 2959$ $\mathrm{s}, 2931 \mathrm{~s}, 2875 \mathrm{~m} ; v_{\mathrm{CH}_{3}}: 1258 \mathrm{~m} ; v_{\mathrm{Si}-\mathrm{C}}: 1173 \mathrm{~m} ; v_{\mathrm{Si}-\mathrm{O}}: 1082 \mathrm{~s}$; $v_{\mathrm{C}-\mathrm{O}-\mathrm{Si}}: 1010 \mathrm{~s}$ (presented in Figures $1 \mathrm{~d}$ and $2 \mathrm{~d}$ ).

Polymerization: Synthesis of OHPS-Cored Star Poly( $\epsilon$-caprolactone). The multifunctional hydroxyl initiator OHPS was placed in a Schlenk flask equipped with a reflux condenser and a magnetic stirrer. $\epsilon$-Caprolactone was added to dissolve the reactant (various $\mathrm{M} / \mathrm{I}$ ratios were used to give different molecular weight). The mixture was heated to 115 ${ }^{\circ} \mathrm{C}$, and then a catalytic amount of $\mathrm{Sn}(\mathrm{Oct})_{2}$ was added. The bulk reaction began to reflux immediately under nitrogen for $24 \mathrm{~h}$. The mixture was then diluted with THF and precipitated into cold methanol to give a high yield of a white crystalline powder. The ${ }^{1} \mathrm{H}$ NMR and FTIR spectra of the synthesized organic/inorganic star PCL are provided in Figures 5b and 2e, respectively.

Preparation of Inclusion Complexes. The eight-arm star PCL was dissolved in acetone. A saturated aqueous solution of $\mathrm{CD}$ was added dropwise to the polymer solution. The mixture was agitated ultrasonically for $30 \mathrm{~min}$ while heating, and then it was left to stand overnight at room temperature. The white powder was collected by centrifugation and then washed with water and acetone to remove any free polymer and uncomplexed CD. Finally, the IC was dried in a vacuum oven at $40{ }^{\circ} \mathrm{C}$ for $48 \mathrm{~h}$.

Characterization. Differential scanning calorimetry (DSC) of the materials was performed under a continuous nitrogen purge $(60 \mathrm{~mL} / \mathrm{min})$ using a TA Instruments DSC 2910 . Each sample was first heated to $200{ }^{\circ} \mathrm{C}$, maintained isothermal for $5 \mathrm{~min}$, and then quickly quenched to $-110{ }^{\circ} \mathrm{C}$. Data were gathered on the second heating cycle at a scan rate of $20^{\circ} \mathrm{C} /$ min over a temperature range from -110 to $200^{\circ} \mathrm{C}$. The glass transition temperature was taken as the midpoint of the heat capacity transition between the upper and lower points of the deviation from the extrapolated glass and liquid lines.

Thermal gravimetric analyses were performed under nitrogen or air using a TA Instruments TGA 2050 thermal gravimetric analyzer at a heating rate of $20^{\circ} \mathrm{C} / \mathrm{min}$ over the range from room temperature to $800{ }^{\circ} \mathrm{C}$. The nitrogen or air flow rate was $60 \mathrm{~mL} / \mathrm{min}$.

Fourier transform IR spectra were obtained using a Nicolet Avatar 320 FT-IR spectrometer; 32 scans were collected with a spectral resolution of $1 \mathrm{~cm}^{-1}$. The conventional $\mathrm{NaCl}$ disk method was employed. A THF solution containing the sample was cast onto a $\mathrm{NaCl}$ disk and dried under conditions similar to those used in the bulk preparation. The holder was placed in the sample chamber, and the spectrum was recorded under a $\mathrm{N}_{2}$ purge to maintain the film's dryness. The weight-average $\left(M_{\mathrm{w}}\right)$ and number-average $\left(M_{\mathrm{n}}\right)$ molecular weights and the polydispersity index $\left(M_{\mathrm{w}} / M_{\mathrm{n}}\right)$ were measured using a Waters 410 gel permeation chromatography (GPC) system equipped with RI and UV detectors and a Styragel columns $(300 \times 7.8$ $\mathrm{mm}$ ). The system was calibrated using polystyrene standards, the eluent was THF, and the flow rate was $1.0 \mathrm{~mL} / \mathrm{min}$.

${ }^{1} \mathrm{H}$ NMR spectroscopic analyses were performed using a Varian Uniytinova-500 NMR spectrometer at $500 \mathrm{MHz}$. All of the spectra were recorded using $\mathrm{CDCl}_{3}$ as the solvent and TMS as the external standard. The ${ }^{13} \mathrm{C}$ CP/MAS NMR spectra were recorded at room temperature using a Bruker DSX-400WB NMR spectrometer with a sample spinning rate of $8.0 \mathrm{kHz}$. The spectra were acquired using a $3.9 \mu$ s proton $90^{\circ}$ pulse, a $1 \mathrm{~ms}$ contact time, and a $3 \mathrm{~s}$ repetition time.

Wide-angle X-ray diffraction (WAXD) spectra were recorded on powdered samples using a Rigaku D/max-2500 type X-ray diffractometer. The radiation source used was Ni-filtered, $\mathrm{Cu}$ $\mathrm{K} \alpha$ radiation at a wavelength of $0.154 \mathrm{~nm}$. The voltage was set at $30 \mathrm{kV}$ and the current at $20 \mathrm{~mA}$. The sample was mounted on a circular sample holder; the proportional counter detector collected data at a rate of $2 \theta=5^{\circ} \mathrm{min}^{-1}$ over the $2 \theta$ range $3-60^{\circ}$.

\section{Results and Discussion}

Synthesis of the POSS-Cored Star PCL. To allow the organic-inorganic hybrid star polymers to be prepared through ring-opening polymerization of lactones, 
Scheme 1. Silylation of Allyl Alcohol with tert-Butyldimethylsilyl Chloride $\left(t-\mathrm{BuMe}_{2} \mathrm{SiCl}\right)$

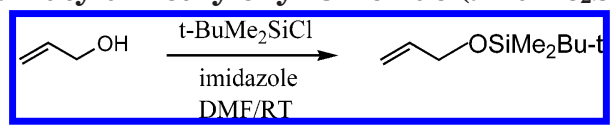

we attached hydroxyl groups to the cubic silsesquioxane. Initially, we attempted to synthesize the octafunctionalized initiator by reacting allyl alcohol with the eight equivalently reactive functional groups of $\mathrm{Q}_{8} \mathrm{M}_{8}{ }^{\mathrm{H}}$. Such a direct hydrosilylation, however, can lead not only to C-silylation but also to O-silylation, ${ }^{38}$ and thus, protective groups must be used to avoid O-silylation. As a protecting group, we chose to use tert-butyldimethylsilyl (TBDMS) chloride because the $\mathrm{TBDMS}^{43}$ protective groups can be readily removed from the parent molecules under mild, neutral reaction conditions by using tetrabutylammonium fluoride at room temperature. ${ }^{42}$ Scheme 1 illustrates the silylation of allyl alcohol with tert-butyldimethylsilyl chloride to give the hydroxylprotected allyl alcohol. The reaction proceeded well in the presence of imidazole at room temperature; we obtained the product in $92 \%$ yield. Scheme 2 summarizes the general approach used to synthesize the OHPS-cored star PCL. Octakis(tert-butyldimethylsiloxylpropyldimethylsiloxy)octasilsesquioxane (OBdsPS) was obtained from the reaction of $\mathrm{Q}_{8} \mathrm{M}_{8}{ }^{\mathrm{H}}$ with the hydroxyl-protected allyl alcohol, which we performed using $\mathrm{Pt}(\mathrm{dvs})$ as the catalyst because it is the most suitable reagent for performing the hydrosilylation. ${ }^{1} \mathrm{H}$ NMR and FT-IR spectra both confirmed that we had produced the desired products, which presents eight tert-butyldimethylsiloxyl "arms" from its cubic. Similar to $\mathrm{Q}_{8} \mathrm{M}_{8}{ }^{\mathrm{H}}$, the OBdsPS possesses eight identical functional groups that have equal reactivity. After removal of the protective groups, we obtained the synthesized OHPS derivative completely substituted with hydroxyl groups, as evidenced by ${ }^{1} \mathrm{H}$ NMR and FT-IR spectroscopies.

All of the polymerization reactions conducted in this work were performed using tin(II) 2-ethylhezanoate [Sn(oct) ${ }_{2}$ ] as the catalyst. $\mathrm{Sn}$ (oct) $)_{2}$ is one of the agents most widely used for initiating the ring-opening polymerization (ROP) of various lactones and lactides. We used the OHPS derivative, which contains eight identical pri-
Table 1. Polymerization of $\epsilon$-Caprolactone with OHPS as Initiator ${ }^{a}$

\begin{tabular}{cccccc}
\hline entry $^{b}$ & $M_{\mathrm{n}}{ }^{c}$ & $M_{\mathrm{w}} / M_{\mathrm{n}}{ }^{d}$ & $T_{\mathrm{m}}\left({ }^{\circ} \mathrm{C}\right)$ & $\Delta H(\mathrm{~J} / \mathrm{g})$ & $\Delta \chi_{\mathrm{cPCL}^{e}}(\%)$ \\
\hline S1 & 1400 & 1.23 & 58 & 67 & 48.2 \\
S2 & 4500 & 1.27 & 58 & 79 & 56.8 \\
S3 & 7800 & 1.31 & 58 & 86 & 61.9
\end{tabular}

${ }^{a}$ The star-shaped poly( $\epsilon$-caprolactone $) \mathrm{S}$ were obtained from ringopening polymerization of $\epsilon$-caprolactone using $\mathrm{Sn}(\text { oct })_{2}$ as a catalyst and OHPS as an initiator. ${ }^{b}$ Samples S1-S3 were polymerized at different $\mathrm{M} / \mathrm{I}$ ratios. ${ }^{c}$ The number-average molecular weights $\left(M_{\mathrm{n}}\right)$ of a single arm were characterized by ${ }^{1} \mathrm{H}$ NMR spectroscopic functional groups analysis. ${ }^{d}$ Weight-average molecular weight $\left(M_{\mathrm{w}}\right) /$ number-average molecular weight $\left(M_{\mathrm{n}}\right)$, as determined by GPC. ${ }^{e} \Delta \chi_{\mathrm{cPCL}}=\Delta H / \Delta H_{0} w$. The melting enthalpy of pure PCL in the completely crystalline state $\left(\Delta H_{0}\right)$ is $139 \mathrm{~J} / \mathrm{g}$ according to the literature. ${ }^{54}$

mary alcohol functionalities, as the initiation center for living ROP of cyclic lactones. $\epsilon$-Caprolactone was reacted with $\mathrm{Sn}(\text { oct })_{2}$ [monomer/catalyst molar ratio $(\mathrm{M} / \mathrm{C})=$ 1000] and OHPS at $115^{\circ} \mathrm{C}$ for $24 \mathrm{~h}$. To obtain poly $(\epsilon-$ caprolactone)s having different molecular weights, we varied the molar ratio (M/I) of $\epsilon$-caprolactone to OHPS. In the FT-IR spectra, we observe that the signal of the hydroxyl groups $\left(3200-3500 \mathrm{~cm}^{-1}\right)$ of the initiator OHPS disappeared, a signal for the carbonyl groups of the star PCL appeared $\left(1733 \mathrm{~cm}^{-1}\right)$, and the characteristic POSS signals were preserved. The compositions and molecular weights of the POSS-cored polymer that we synthesized were characterized by ${ }^{1} \mathrm{H}$ NMR spectroscopy (Table 1). We determined the molecular weight distributions by GPC, but we note that the molecular weights are not absolute values because of hydrodynamic volumes of the star polymers differ from those of the linear PS standards we used for calibration. The molecular weights of a single PCL arm (entries S1-S3) ranged from 1200 to 7800 with $M_{\mathrm{w}} / M_{\mathrm{n}}$ ratios between 1.2 and 1.3. We varied the relative and overall lengths of the PCL arms to investigate the effect that the inorganic-organic composition had on the formation of the polymer inclusion complexes with CDs.

Formation of the Inclusion Complexes. It has been found ${ }^{23}$ that the cross-sectional areas of polymers are closely correlated to the cavity size of the CDs with which they form inclusion complexes. $\alpha-C D$ and $\gamma-C D$ form ICs with linear PCL of various molecular weights

Scheme 2. Synthesis of Octakis(tert-butyldimethylsiloxylpropyldimethylsiloxy)octasilsesquioxane, Octakis(3-hydroxypropyldimethylsiloxy)octasilsesquioxane, and the OHPS-Cored Eight-Arm PCL Star Polymers

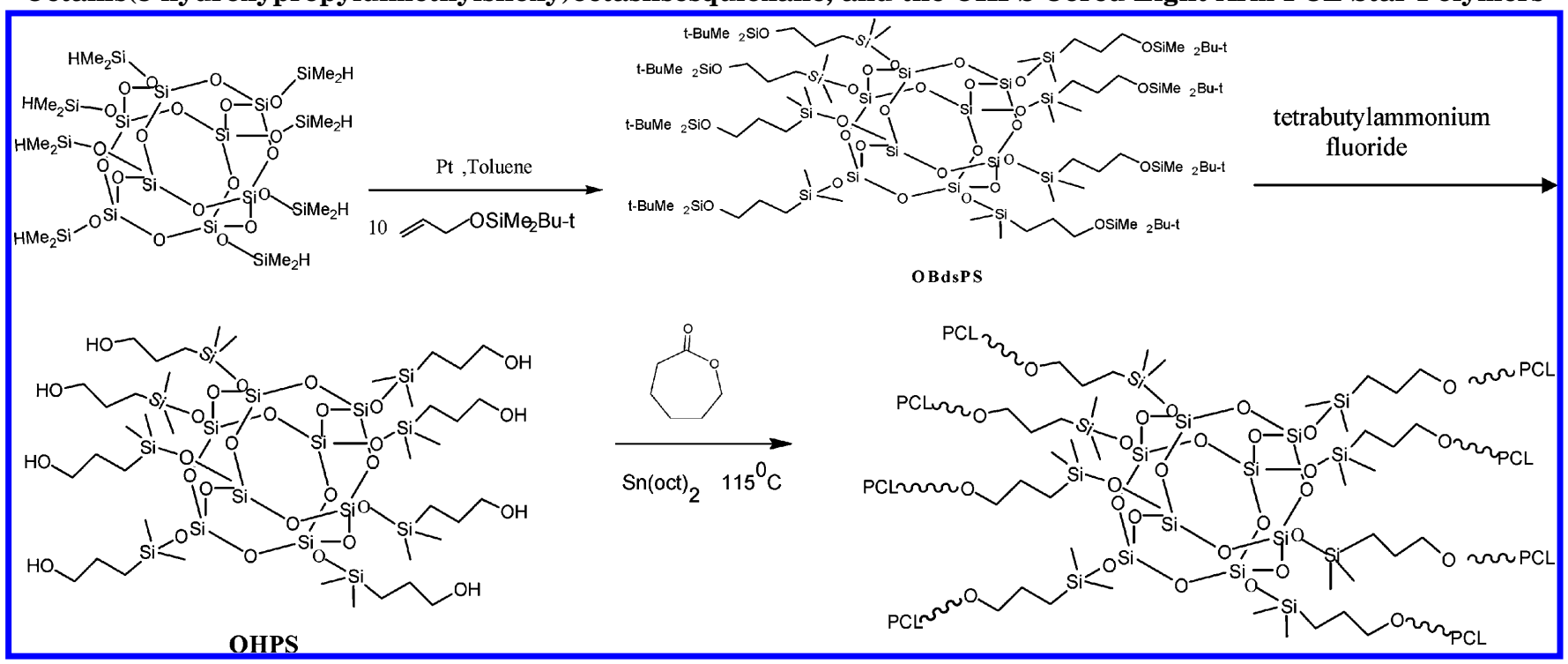


Scheme 3. Proposed Structures of $\alpha$-CD/PCL ICs (Upper) and $\gamma$-CD/PCL ICs (Bottom)

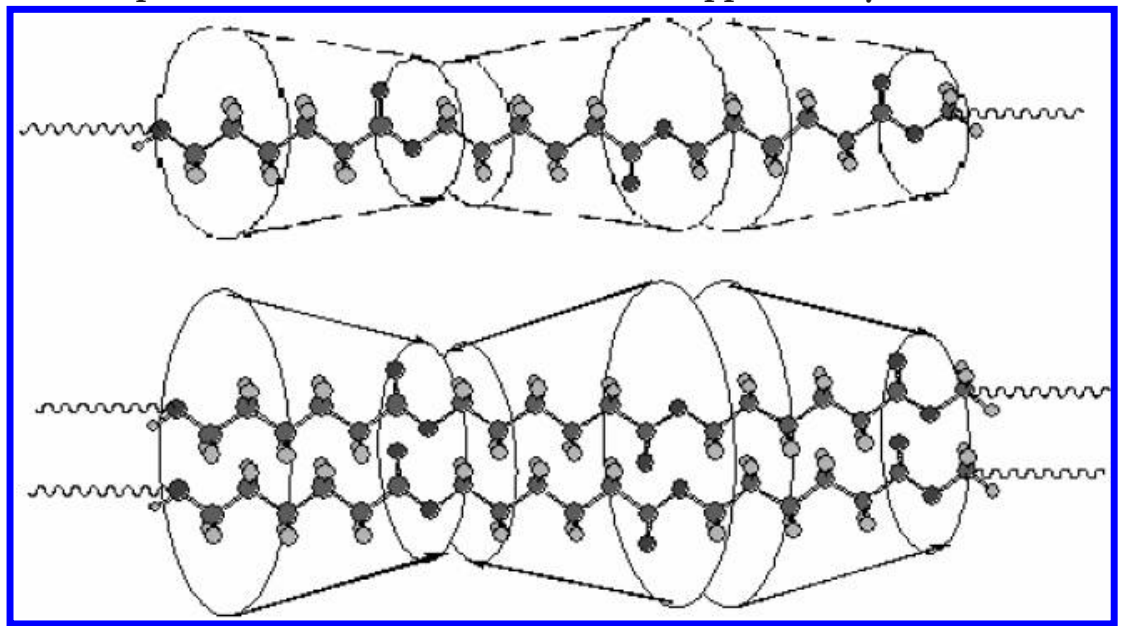

to give crystalline compounds in high yield, whereas the $\beta$-CD complexes with linear PCL are formed only in moderate yield. ${ }^{41}$ This situation arises because the PCL polymer chains in either their all-trans or kinked conformations are slim enough to fit properly into the $\alpha-C D$ cavities, but twisting of these polymer chains is required if they are fill the cavities of $\beta$-CD. ${ }^{44}$

The inclusion complexes formed between linear PCL and CDs have been studied on a number of occasions. ${ }^{41,44,45}$ The stoichiometry of the ICs is $1: 1(\epsilon-\mathrm{CL}$ unit to CD) for the $\alpha$-CD/PCL IC and 2:1 for the $\gamma-\mathrm{CD} /$ PCL ICs; i.e., the $\alpha$-CD/PCL IC contains a single chain in each $\alpha$-CD channel while the $\gamma$-CD/PCL IC contains two adjecent PCL chains, as illustrated in Scheme $3 .^{28}$ When we added aqueous solutions of $\alpha-C D$ and $\gamma-C D$ to solutions of the synthesized organic/inorganic hybrid star PCLs (S1, S2, S3) at a temperature above the melting point of each PCL, all of the solutions gradually turned turbid, indicating the formation of crystalline inclusion complexes between the polymers and the CDs. ${ }^{46}$ Similar to their linear chain analogues, these multiarm PCLs did not form inclusion complexes with $\beta$-CD. We isolated the gellike precipitates of these complexes by centrifugation and washed them with water to remove any uncomplexed $\mathrm{CD}$ and then with tetrahydrofuran (THF) to remove any free star PCL.

The crystal structures of the inclusion complexes were established by XRD. Columnar structures are normally assigned to the ICs of linear chain polymers with CDs. Figure 3 displays the XRD patterns of $\alpha-\mathrm{CD}, \alpha-\mathrm{CD} / \mathrm{S} 1$ $\mathrm{IC}$, and $\gamma$-CD, $\gamma$-CD/S1 IC. The diffraction pattern of the pure $\mathrm{S} 1$ possesses very strong crystalline diffraction peaks at $21.3^{\circ}$ and $23.6^{\circ}$ from the PCL segments. The patterns of these two synthesized ICs are substantially different from those of the pure S1, $\alpha-C D$, and $\gamma-C D$; obviously, they are not merely a simple superposition of their respective IC components. Figure 3a displays the XRD patterns of $\alpha-C D$ and $\alpha-C D / S 1$ IC. The peaks corresponding to the PCL crystalline phase have disappeared and a new strong peak appears at $2 \theta=20^{\circ}$, which is typical for $\alpha$-CD/polymer ICs. ${ }^{47,48}$ Similar patterns are presented for the $\alpha-\mathrm{CD} / \mathrm{S} 2$ and $\alpha-\mathrm{CD} / \mathrm{S} 3$ ICs. The appearance of the characteristic peak at $2 \theta=$ $20^{\circ}$ confirms that columnar structures did indeed form for the complexes between $\alpha-C D$ and the $\mathrm{S}$ series, rather than cagelike structures. ${ }^{28}$

Figure $3 \mathrm{~b}$ displays the XRD patterns of $\gamma$-CD and the $\gamma$-CD/S1 IC. The pattern of the $\gamma$-CD/S1 IC is also different from that of pure $\gamma$-CD, in which the CDs exists in a cage-type packing arrangement. ${ }^{49}$ The fingerprint peak for the columnar structure of the $\gamma-\mathrm{CD} /$

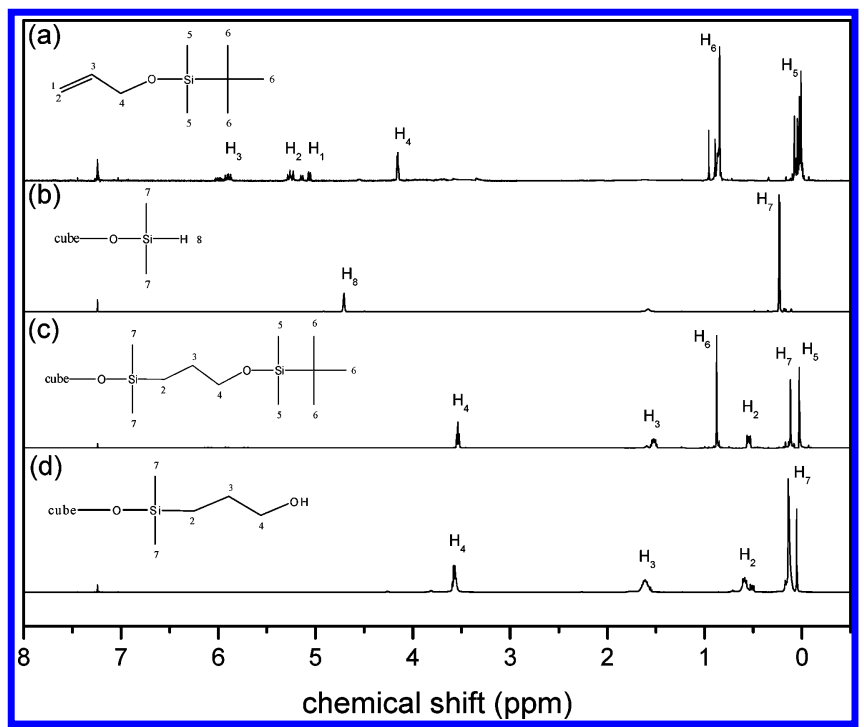

Figure 1. ${ }^{1} \mathrm{H}$ NMR spectra of (a) silylated allyl alcohol, (b) $\mathrm{Q}_{8} \mathrm{M}_{8} \mathrm{H}$, (c) octakis $($ tert-butyldimethylsiloxylpropyldimethylsiloxy)octasilsesquioxane, and (d) OHPS.

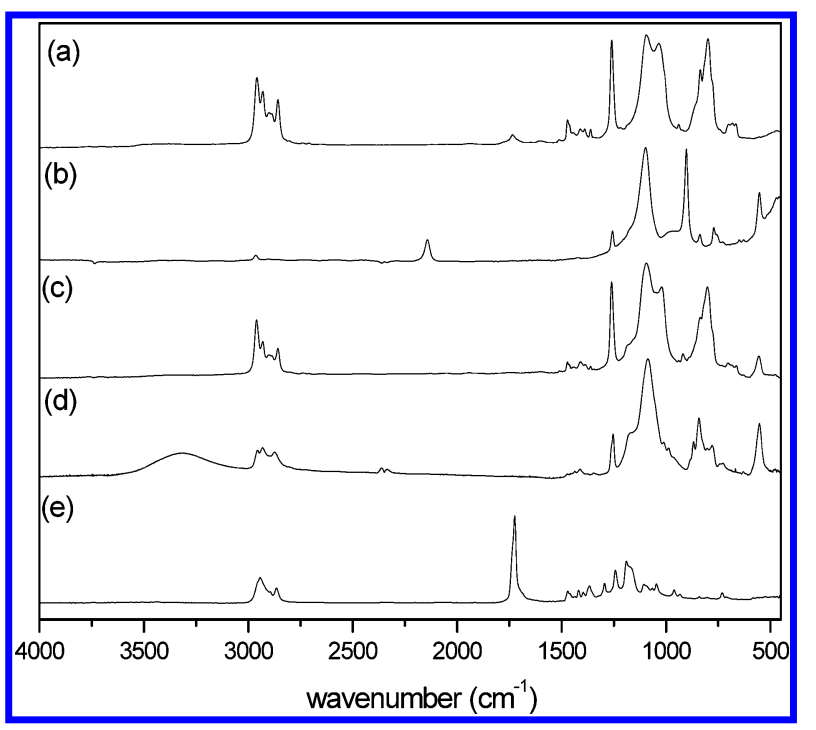

Figure 2. FT-IR spectra of (a) silylated allyl alcohol, (b) $\mathrm{Q}_{8} \mathrm{M}_{8} \mathrm{H}$, (c) octakis(tert-butyldimethylsiloxylpropyldimethylsiloxy)octasilsesquioxane, (d) OHPS, and (e) the OHPS-cored eight-arm PCL star polymer. 


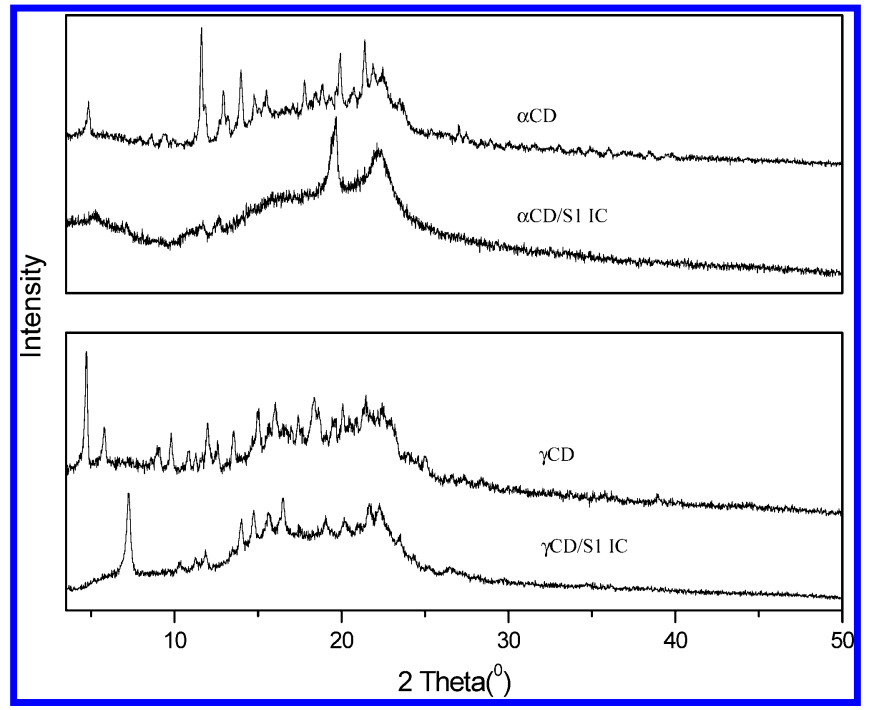

Figure 3. ${ }^{1} \mathrm{H}$ NMR spectra of (a) $\alpha$-CD, (b) the synthesized star PCL (S3: $\left.M_{\mathrm{n}}=7800\right)$, and (c) the $\alpha-\mathrm{CD} / \mathrm{S} 3$ IC.

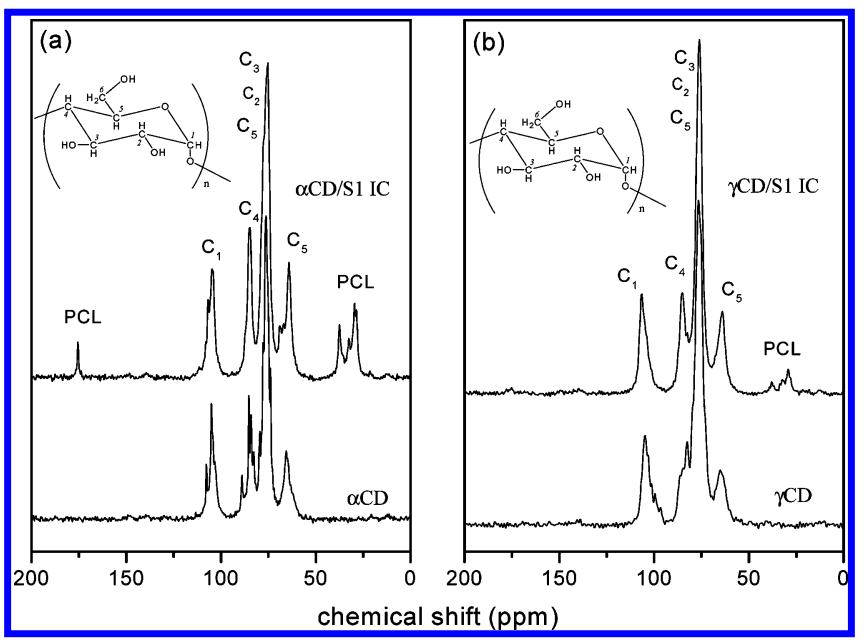

Figure 4. ${ }^{13} \mathrm{C} \mathrm{CP} / \mathrm{MAS}$ NMR spectra of (a) $\alpha-\mathrm{CD}$ and the $\alpha-\mathrm{CD} / \mathrm{S} 1 \mathrm{IC}$ and (b) $\gamma-\mathrm{CD}$ and the $\gamma-\mathrm{CD} / \mathrm{S} 1 \mathrm{IC}$.

polymer ICs at $2 \theta=8^{\circ}$ is present in the XRD patterns of the $\gamma$-CD/S1 IC and the other two $\gamma$-CD complexes; this observation suggests that the three complexes also have columnar structures ${ }^{24}$ These results indicate that the star polymer formed ICs with both $\alpha$-CD and $\gamma$-CD that have columnar structures in which almost all of the PCL chains of the star polymer are included within $\alpha-C D$ or $\gamma$-CD channels. One or two PCL chains are isolated from their environment by their surrounding $\alpha$-CD or $\gamma$-CD molecules, respectively; consequently, PCL is unable to aggregate to form any crystalline phase other than that of the newly generated IC. We obtained more convincing evidence for IC formation from the solid-state ${ }^{13} \mathrm{C}$ CP/MAS NMR spectra presented in Figure 4.

Figure 4 a displays the solid-state ${ }^{13} \mathrm{C}$ NMR CP/MAS NMR spectra of $\alpha-C D$ and $\alpha-C D / S 1$ IC; the spectrum of the $\alpha$-CD presents resolved resonances for the carbon nuclei of the glucose units, whereas the spectrum of the $\alpha-\mathrm{CD} / \mathrm{S} 1 \mathrm{IC}$ displays unresolved resonances. The spectrum of $\alpha$-CD presents multiple lines because of the $C(1)$ and $\mathrm{C}(4)$ resonances from each of the six $\alpha$-1,4-linked glucose residues. ${ }^{18}$ On the other hand, the resonances of the C(1) and C(4) nuclei of the $\alpha-C D / S 1$ IC appear as sharp singlets, which indicate that the $\alpha$-CD units adopt symmetric cyclic confirmations upon complexation such

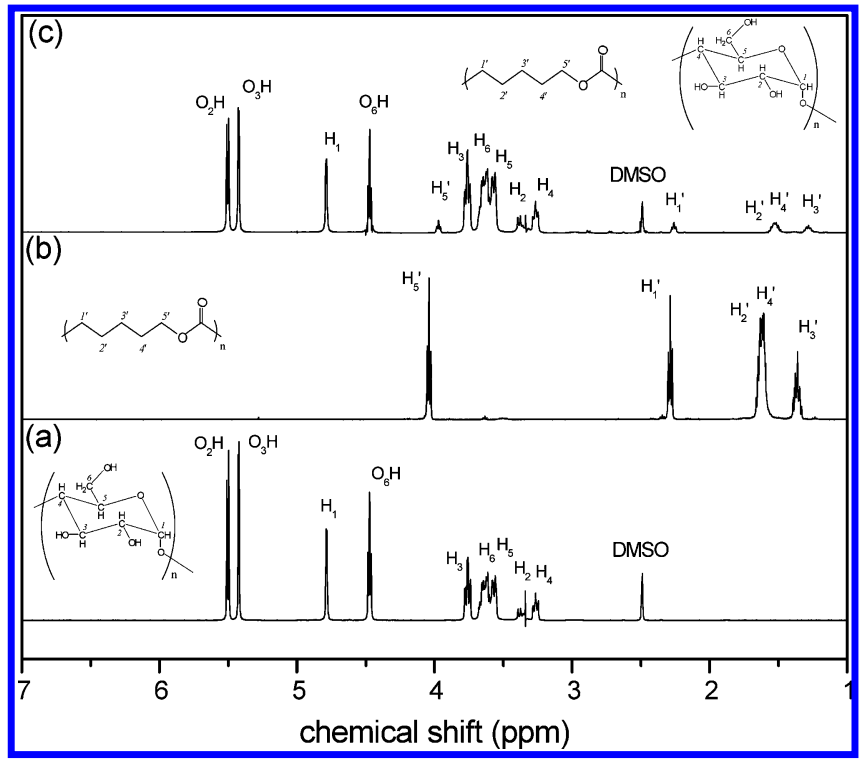

Figure 5. X-ray diffraction patterns of (a) $\alpha-\mathrm{CD}$ and the $\alpha$-CD/ S1 IC and (b) $\gamma-\mathrm{CD}$ and the $\gamma-\mathrm{CD} / \mathrm{S} 1 \mathrm{IC}$.

that each glucose unit exists in a similar environment. ${ }^{47,50}$ In addition, the chemical shifts for the PCL in the inclusion complex are similar to those observed in the semicrystalline sample, suggesting that the PCL adopts an extended all-trans conformation in the $\alpha-C D$ channels. ${ }^{28}$ The spectra of the other two $\alpha$-CD complexes display similar results. Similar observations, which we believe support the formation of ICs between CDs and polymers, have been made from the solid-state ${ }^{13} \mathrm{C} N M R$ CP/MAS NMR spectra of ICs prepared form a number of other polymers and a range of CDs. ${ }^{23,46}$ Figure $4 \mathrm{~b}$ presents the solid-state ${ }^{13} \mathrm{C}$ NMR CP/MAS NMR spectra of $\gamma$-CD and the $\gamma$-CD/S1 IC. The spectrum of the complex indicates that the CD adopts a symmetrical cyclic conformation in the complex, whereas $\gamma$-CD assumes a less-symmetrical conformation in the crystal when it does not include a guest in its cavity. ${ }^{50}$ Our results for the other two ICs were similar, and they are all consistent with the results reported previously for $\gamma$-CD/linear PCL ICs. ${ }^{28}$ The ${ }^{13} \mathrm{C}$ NMR CP/MAS NMR spectra of the complexed and uncomplexed CDs are consistent with the results of our XRD studies. Therefore, we believe that the PCL chains of the synthesized star polymers are included within the cavities of the CDs in columnar form.

We established the compositions of the inclusion complexes by ${ }^{1} \mathrm{H}$ NMR spectroscopy. Figure 5 displays the ${ }^{1} \mathrm{H}$ NMR spectra of pure $\alpha-\mathrm{CD}, \mathrm{S} 1$, and the inclusion complex formed between $\alpha-C D$ and $S 1$. The latter spectrum (Figure 5c) displays peaks corresponding to both the $\alpha-C D$ and the star PCL S1, which are presented in parts $\mathrm{a}$ and $\mathrm{b}$ of Figure 5, respectively. When comparing the integral of a peak for $\alpha-C D(1 \mathrm{H})$ with that of the PCL methylene groups, we find that on average 1.9 monomer units of $\epsilon$-CL bind to one $\alpha$-CD molecule. On the other hand, the average numbers of $\epsilon$-CL repeat units bound to one $\gamma$-CD molecule in the $\gamma$-CD/S1 IC was 3.3. Again, we note that the stoichiometry for $\alpha$-CD/linear PCL ICs is 1:1 ( $\epsilon$-CL monomer unit: CD) and 2:1 for $\gamma$-CD/linear PCL ICs. ${ }^{28}$ Our results imply that these inclusion complexes are not formed in perfect stoichiometry. Table 2 lists the results for all of the CD/S ICs. All of the $\epsilon-\mathrm{CL} / \alpha-\mathrm{CD}$ ratios $(1.3-1.9)$ for the $\alpha-\mathrm{CD} / \mathrm{S}$ ICs are $>1$, which suggests that some of the 
Table 2. Stoichiometries (CL unit/CDs) of Inclusion Complexes

\begin{tabular}{|c|c|c|c|}
\hline entry & $M_{\mathrm{n}}$ & $\begin{array}{c}\text { stoichiometry }^{a} \\
\text { (PCL:CD) of } \alpha-C D / \text { star } \\
\text { PCL ICs }\end{array}$ & $\begin{array}{c}\text { stoichiometry }^{a} \\
\text { (PCL:CD) of } \gamma \text {-CD/star } \\
\text { PCL ICs }\end{array}$ \\
\hline S1 & 1200 & 1.9 & 3.1 \\
\hline S2 & 4500 & 1.6 & 2.7 \\
\hline S3 & 7800 & 1.3 & 2.2 \\
\hline
\end{tabular}

${ }^{a}$ The stoichiometry of each star PCL/CD ICs was determined by ${ }^{1} \mathrm{H}$ NMR spectroscopy.

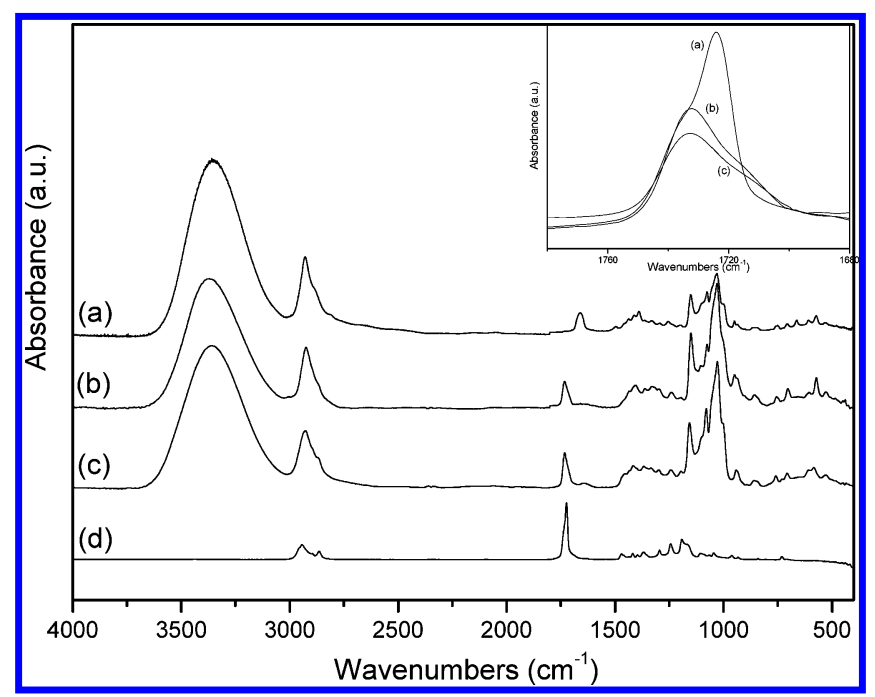

Figure 6. FT-IR spectra of (a) $\alpha-C D$, (b) $\alpha-C D / S 1$ IC, (c) $\gamma-C D /$ S1 IC, and (d) and pure S1. The inset presents an expansion of the carbonyl absorption region of (a) pure $\mathrm{S} 1$, (b) $\alpha-\mathrm{CD} / \mathrm{S} 1$ IC, and (c) $\gamma$-CD/S1 IC.

$\epsilon$-CL units are free from inclusion. In the case of the ICs formed from $\gamma$-CD, the ratios are between 2.2 and 3.1, which indicates, again, that some of the $\epsilon$-CL units are not included within the CD channels. In the synthesized organic/inorganic star-shaped PCL, the POSS core acts as the linking point for the eight PCL chains. We envision that a few of the $\epsilon$-CL units near the bulky POSS core of the eight-arm star PCL have difficulty to be included within CD units because of the steric hindrance provided by the core moiety. This effect leads to stoichiometries higher than 1:1 and 2:1 for the $\alpha$ - and $\gamma$-CD ICs, respectively, especially when the molecular weight of the polymer is low, such as for S1. The effect of steric hindrance is clearly evident in Table 2 where we note that the ICs of S1 with $\alpha$-CD and $\gamma-C D$ provided much higher stoichiometries than did the other ICs.

We obtained further strong evidence for the inclusion complexation from the FT-IR spectra of the $\alpha-C D, \alpha-C D /$ S1 IC, $\gamma$-CD/S1 IC, and pure S1 (Figure 6). The spectra of the ICs display signals of both $\mathrm{S} 1$ and the CDs, which indicates that both these species are present in the inclusion complexes. The broad hydroxyl band of $\alpha-C D$ at ca. $3360 \mathrm{~cm}^{-1}$ is shifted to $3390 \mathrm{~cm}^{-1}$ in the spectrum of the IC. This shift can be ascribed to the noncovalent interactions of the hydroxyl groups of pairs of $\alpha-C D$ units and between $\alpha-C D$ and the included PCL backbone. The most distinct bands in the spectra of the ICs are those at $1733 \mathrm{~cm}^{-1}$, which are due to the stretching of the carbonyl groups of PCL. The carbonyl absorption band of pure $\mathrm{S} 1$ is well resolved into a peak at 1726 $\mathrm{cm}^{-1}$ and a prominent shoulder at $1736 \mathrm{~cm}^{-1}$; according to a previous report on semicrystalline PCL, these signals correspond to the carbonyl absorptions of the

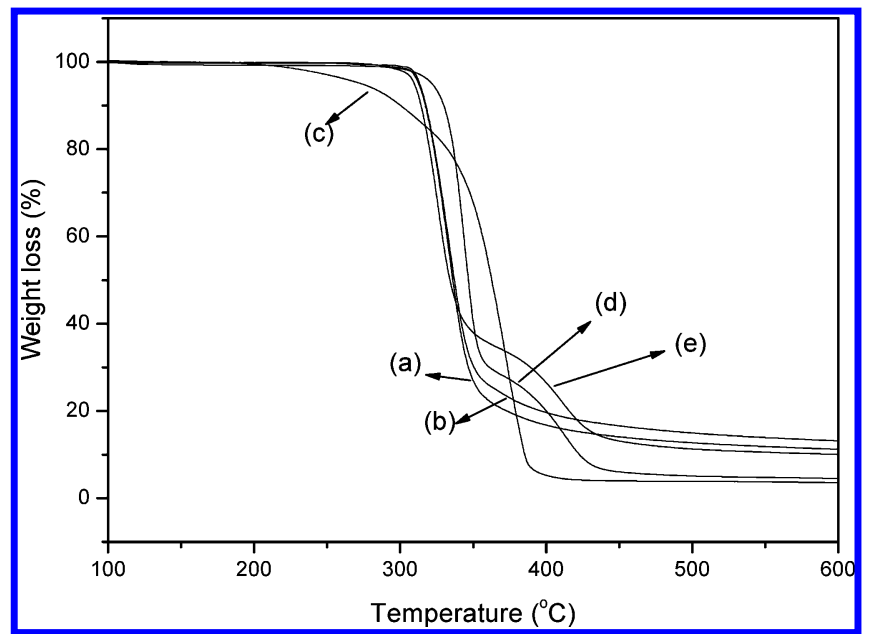

Figure 7. TGA curves obtained at a heating rate of $20{ }^{\circ} \mathrm{C} /$ min under a nitrogen atmosphere for (a) $\alpha-C D$, (b) $\gamma-C D$, (c) S1, (d) $\alpha-C D / S 1$ IC, and (e) $\gamma-C D / S 1$ IC.

crystalline and amorphous PCL phases. ${ }^{51}$ In the complexed form, we believe that these PCL chains are located and confined within the IC channels, and thus, they are unable to aggregate to form PCL crystals; i.e., the peak at $1726 \mathrm{~cm}^{-1}$ is depressed in the spectrum of ICs. ${ }^{52,53}$ The inset of Figure 6 presents an expansion of the carbonyl region of the spectra of pure S1, the $\alpha-\mathrm{CD} /$ S1 IC, and the $\gamma$-CD/S1 IC. When compared with the spectrum of pure S1 (inset), the spectra of the ICs indicate that the carbonyl absorption of the crystalline PCL region $\left(1726 \mathrm{~cm}^{-1}\right)$ completely disappeared, and only the carbonyl absorption of noncrystalline PCL chains $\left(1736 \mathrm{~cm}^{-1}\right)$ can be detected. The results obtained form these FT-IR spectra are consistent with those obtained from the XRD and solid-state ${ }^{13} \mathrm{C}$ CP/MAS NMR spectra; i.e., no crystalline PCL phase exists in either of these two ICs. Most of the units of the PCL chains are included within the channels of the $\alpha-\mathrm{CDs}$ and $\gamma$-CDs.

From DSC measurements of the synthesized POSScor star PCL, we observed a strong melting peak for the PCL parts (not shown here). The crystallinity ${ }^{54}\left(\Delta \chi_{\mathrm{cPCL}}\right)$ of the PCL samples can be calculated on the basis of its enthalpy of fusion as determined by DSC measurements. Table 1 summarizes the results: $\mathrm{S} 1$ has a value of $\Delta \chi_{\mathrm{cPCL}}$ of ca. 49 , while that of S3 is higher at (ca. 62). This finding is reasonable because the decrease in crystallinity of the star PCL is due to the stiffening and binding effects exerted by the introduction of the bulky POSS core. Therefore, a higher fraction of POSS in the POSS-core star PCL results in lower crystallinity. On the other hand, we observed no fusion peak for any of the ICs investigated in the heating run (data not shown); this finding indicates that the original crystalline polymer phase is missing in the complex. This result further confirms that the PCL chains are indeed included within CD channels upon the formation of the CD ICs. ${ }^{45}$

These inclusion complexes are thermally stable. Figure 7 displays the results of the TGA measurements recorded at up to $600{ }^{\circ} \mathrm{C}$ for the pure $\alpha$-CD, $\gamma$-CD, S1 and the $\alpha-\mathrm{CD} / \mathrm{S} 1$, and $\gamma-\mathrm{CD} / \mathrm{S} 1 \mathrm{ICs}$. These ICs undergo two-step thermal degradation. The first step can be attributed mainly to decomposition of the CDs, while the second step is mainly that of the star PCLs. The onset decomposition temperatures of S1 and $\alpha-\mathrm{CD}$ in the $\alpha-\mathrm{CD} / \mathrm{S} 1 \mathrm{IC}$ are observed at 325 and $382^{\circ} \mathrm{C}$, which 
Scheme 4. Proposed Structures of the $\alpha$-CD/Eight-arm Star PCL ICs (Left) and the $\gamma$-CD/Eight-Arm Star PCL ICs (Right), Emphasizing the Formation of Columnar Structures Possessing One and Two Arms, Respectively

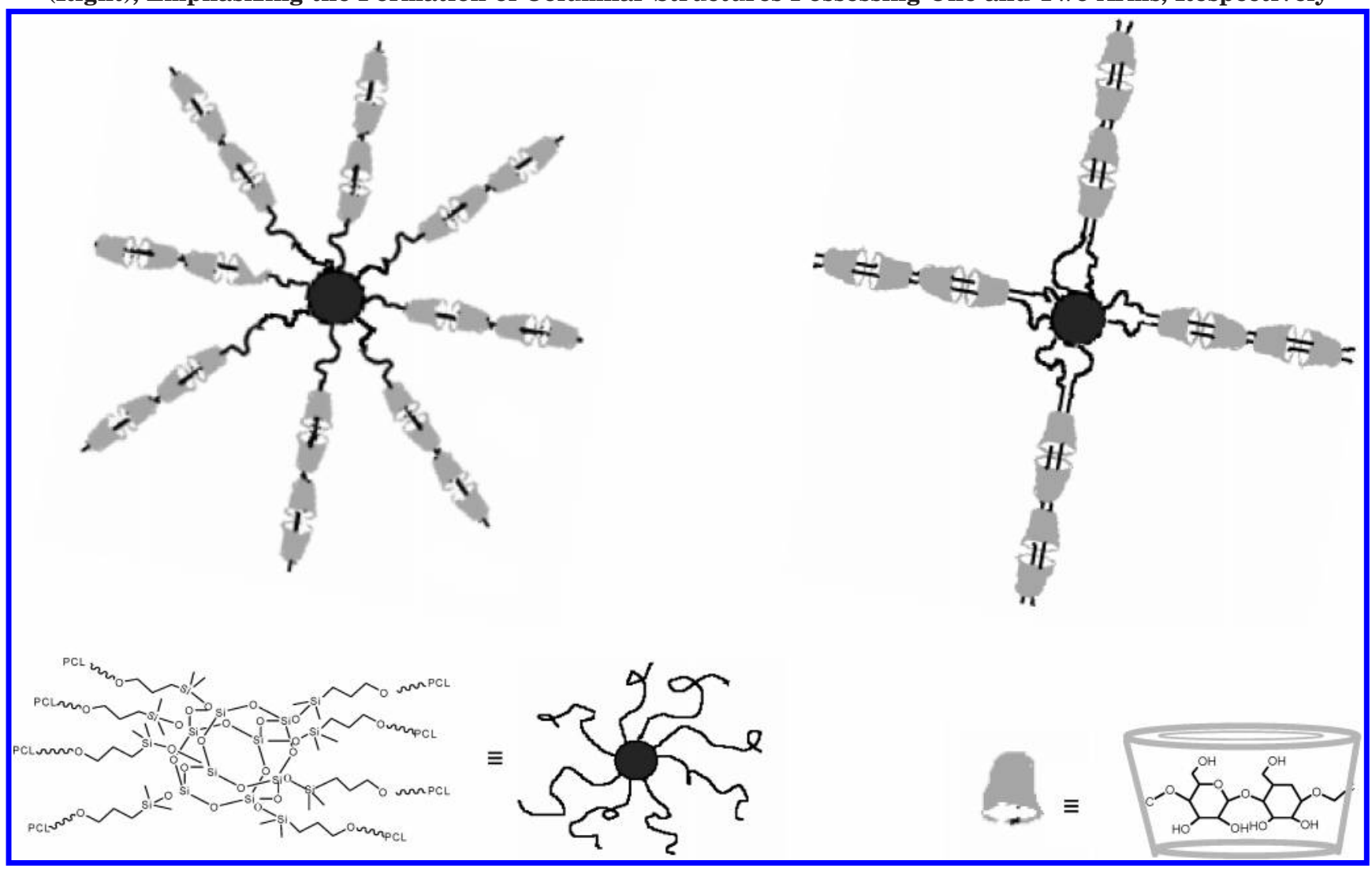

Scheme 5. Proposed Lightly Physically Crosslinked Network Structure of the $\gamma$-CD/Star-PCL Inclusion Complex

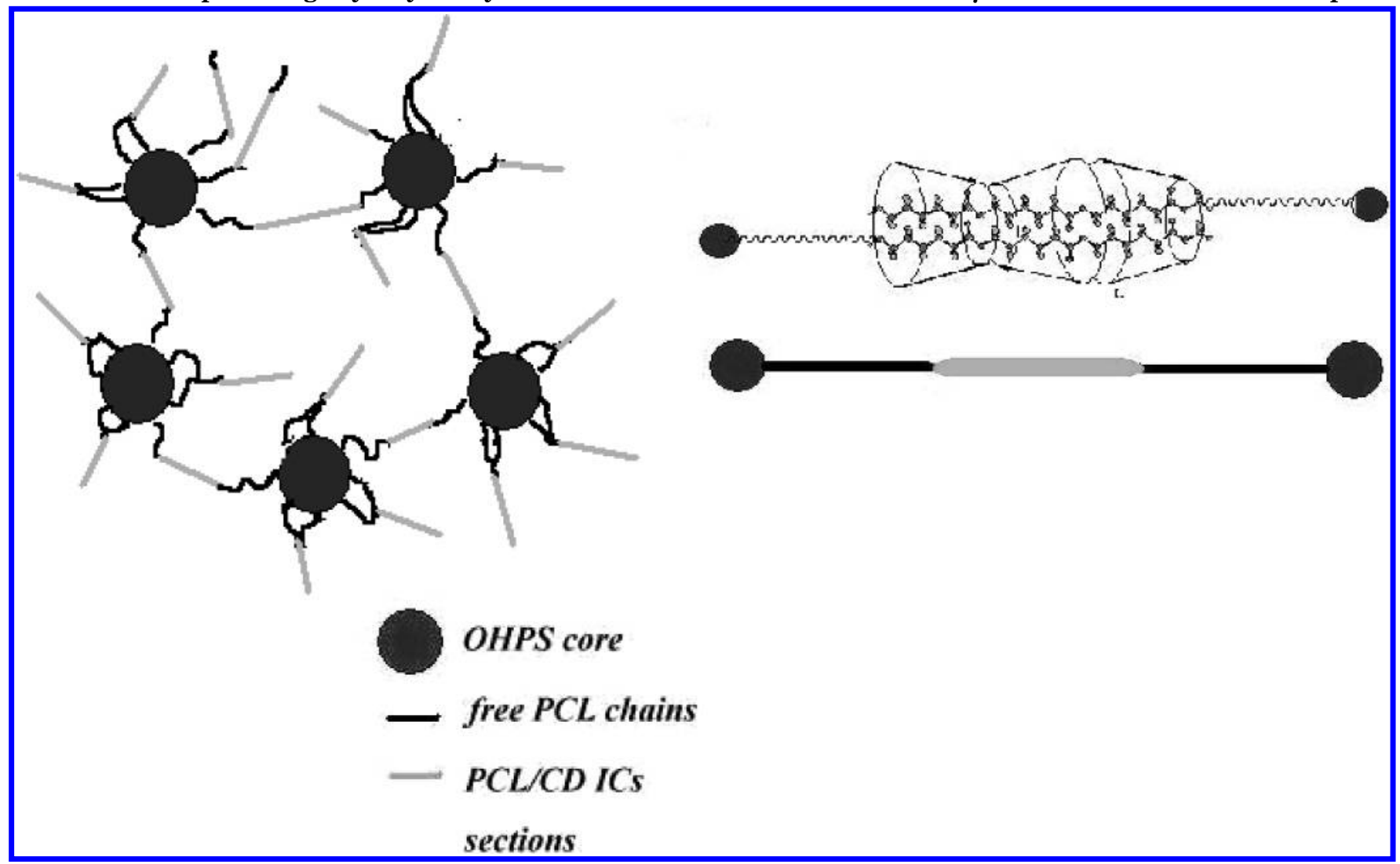

are higher than those of the pure S1 and $\alpha-\mathrm{CD}$. We observe similar results for the $\gamma$-CD/S1 IC and the other CD ICs. ${ }^{55}$ Table 3 summarizes the initial decomposition temperatures of the free and complexed CDs and star-
PCL polymers. All of the star polymers in ICs have decomposition temperatures higher than that of the pure polymer (shift from ca. 360 to $380{ }^{\circ} \mathrm{C}$ ), which indicates that complexation with the $\mathrm{CD}$ enhances the 
Table 3. Summary of the Thermal Characteristics of the CDs, the Synthesized Star PCL, and Their Inclusion Complexes

\begin{tabular}{|c|c|c|c|c|c|c|c|}
\hline \multirow[b]{2}{*}{ entry } & \multicolumn{3}{|c|}{$T_{\mathrm{d}, \text { free }}{ }^{a}\left({ }^{\circ} \mathrm{C}\right)$} & \multicolumn{2}{|c|}{$T_{\mathrm{d}, \text { complexes }}{ }^{b}\left({ }^{\circ} \mathrm{C}\right)$} & \multicolumn{2}{|c|}{$T_{\mathrm{d}, \text { complexes }}{ }^{b}\left({ }^{\circ} \mathrm{C}\right)$} \\
\hline & $\alpha-C D$ & $\gamma-\mathrm{CD}$ & star-PCL & $\alpha-C D$ & star-PCL & $\gamma-\mathrm{CD}$ & star-PCL \\
\hline $\mathrm{S} 1$ & 313 & 314 & 360 & 325 & 382 & 312 & 380 \\
\hline S2 & 313 & 314 & 362 & 324 & 384 & 311 & 381 \\
\hline S3 & 313 & 314 & 365 & 327 & 384 & 315 & 383 \\
\hline
\end{tabular}

${ }^{a}$ The values of $T_{\mathrm{d}}$ for the free CDs and each component of the star PCL were determined from TGA curves as the point at which 5\% mass loss occurred at each degradation step. ${ }^{b}$ The values of $T_{\mathrm{d}}$ for each component in the star-polymer/CDs were determined from TGA curves as the point at which 5\% mass loss occurred at each degradation step.

thermal stability of the star PCLs. The thermal stabilities of the CDs in their ICs are also higher than those of the pure CDs, which implies that the presence of guests included within the CD channels can improves the thermal stability of the original CDs.

According to the stoichiometries ratios $(\epsilon$-CL unit:CD) that we determined by ${ }^{1} \mathrm{H}$ NMR spectroscopy, we believe that the $\alpha-C D /$ star PCL ICs contain single PCL chains in each $\alpha-C D$ channel while the $\gamma-\mathrm{CD} /$ star PCL ICs contain two PCL chains; i.e., they possess similar architectures to those found in the frameworks of the corresponding CD/linear PCL ICs. Scheme 4 illustrates some possible structures for the inclusion complexes formed between the star PCLs and $\alpha-\mathrm{CD} / \gamma-\mathrm{CD}$, with eight- and four-rod arms attached to the POSS core, respectively. Another possible structure for the $\gamma-\mathrm{CD} /$ star PCL ICs might form as a result of $\gamma$-CD containing two PCL chains simultaneously within the one channel. The inclusion of arms from two different star PCLs within the same $\gamma$-CD IC column may occur as a result of the unequal splaying of each arm of the star polymer. As a result, a gellike lightly physically cross-linked structure would form. Scheme 5 presents a proposed structure for this cross-linked $\gamma$-CD/star PCL IC where the POSS core would be the only uncomplexed material located between the CD IC crystalline cross-links.

\section{Conclusions}

We have synthesized a series of organic-inorganic hybrid star PCLs through coordinated ring-opening polymerization of $\epsilon$-caprolactone using POSS as the initiator. Similar findings were reported previously for linear PCL analogues; the star PCL forms inclusion complexes with $\alpha$ - and $\gamma$-CD, but not with $\beta$-CD. We characterized the CD ICs using various analytical methods. From the results of XRD and solid-state ${ }^{13} \mathrm{C}$ CP/MAS NMR spectroscopic analyses, when the star PCLs were confined within the CD channels, they lost their original crystalline properties and formed columnar crystalline structures. The stoichiometries (PCL:CD) for all of the ICs with $\alpha$ - and $\gamma$-CD that we investigated here are greater than those of the corresponding CD/ linear PCL ICs because of the steric hindrance around the bulky POSS core, which causes some of the $\epsilon$ caprolactone units near the core to be free from forming the ICs. FT-IR spectroscopic and DSC measurements further confirmed that these PCL polymer chains are indeed included within the channels provided by the CDs. According to our results, we have proposed a number of possible structures for the CD/star PCL ICs in which the rodlike arms are attached to the POSS core. For the $\gamma$-CD/star PCL ICs, one possible structure that may form consists of a very lightly physically crosslinked network in which the POSS core behaves as a cross-linker.
Acknowledgment. The authors like thank the National Science Council, Taiwan, Republic of China, for financially supporting this research.

\section{References and Notes}

(1) Harada, A.; Li, J.; Kamachi, M. Nature (London) 1993, 364, 516.

(2) Lo Nostro, P.; Lopes, J. R.; Ninham, B. W.; Baglioni, P. J. Phys. Chem. B 2002, 106, 2166.

(3) Okada, M.; Takashima, Y.; Harada, A. Macromolecules 2004, 37,7075 .

(4) Liu, Y.; Zhao, Y. Li.; Zhang, H. Y.; Li, X. Y.; Liang, P.; Zhang, X. Z.; Xu, J. J. Macromolecules 2004, 37, 6362-6369.

(5) Wenz, G. Angew. Chem., Int. Ed. Engl. 1994, 33, 803.

(6) Rusa. C. C.; Bullions, T. A.; Fox, J.; Porbeni, F. E.; Wang, X. Tonelli, A. E. Langmuir 2002, 18, 10016.

(7) Fujita, H.; Ooya, T.; Yui, N. Macromolecules 1999, 32, 2534.

(8) Ceccato, M.; Lo Nostro, P.; Baglioni, P. Langmuir 1997, 13, 2436.

(9) Harada, A.; Li, J.; Kamachi, M. Nature (London) 1994, 370, 126.

(10) Harada, A.; Kamachi, M. Macromolecules 1990, 23, 2821.

(11) Shuai, A.; Porbeni, F. E.; Wei, M.; Shin, D.; Tonelli, A. E. Macromolecules 2001, 34, 7355 .

(12) Okada, M.; Kawaguchi, Y.; Okumura, H.; Kamachi, M.; Harada, A. J. Polym. Sci. Part A: Polvm. Chem. 2000, 38, 4839.

(13) Li, X.; Li, J.; Leong, K. W. Macromolecules 2003, 36, 1209.

(14) Ikeda, T.; Lee, W. K.; Ooya, T.; Yui, N. J.Phvs. Chem. B 2003, 107,14

(15) Jiao, H.; Goh, S. H.; Valiyaveettil, S. Macromolecules 2003 , 36,4241

(16) Zhu, X.; Chen, L.; Yan, D.; Chen, Q.; Yao, Y.; Xiao, Y.; Hou, J.; Li, J. Langmuir 2004, 20, 484.

(17) Wei, M.; Bullions, T. A.; Rusa, C. C.; Wang, X.; Tonelli, A. E. J. Polvm. Sci. Part B: Polvm. Phys. 2004, 42, 386.

(18) Harada, A. Coord. Chem. Rev. 1996, 148, 115.

(19) Harada, A.; Li, J.; Kamachi, M. Macromolecules 1993, 26, 5698.

(20) Harada, A.; Li, J.; Kamachi, M. J. Chem. Soc., Chem. Commun. 1990, 1322

(21) Harada, A.; Okada, M.; Li, J.; Kamachi, M. Macromolecules 1995, 28,8406

(22) Harada, A.; Li, J.; Kamachi, M. Chem. Lett. 1993, 237.

(23) Harada, A.; Suzuke, S.; Okada, M.; Kamachi, M. Macromolecules 1996, 29, 5611 .

(24) Harada, A.; Nishiyama, T.; Kawaguchi, Y.; Okada, M.; Kamachi, M. Macromolecules 1997, 30, 7115.

(25) Shuai, X.; Porbeni, F. E.; Wei, M.; Bullions, T.; Tonelli, A. E. Macromolecules 2002, 35, 3778.

(26) Lu, J.; Mirau, P. A.; Tonelli, A. E. Macromolecules 2001, 34, 3276.

(27) Wei, M.; Davis, W.; Uraban, B.; Song, Y.; Porbeni, F. E.; Wang, X.; White, J. L.; Balik, C. M.; Rusa, C. C.; Fox, J.; Tonelli, A. E. Macromolecules 2002, 35, 8039.

(28) Huang, L.; Allen, E.; Tonelli, A. E. Polvmer 1998, 39, 4857.

(29) Nasanthan, N.; Tonelli, A. E.; Nojima, S. Macromolecules 1994, 27, 7220 .

(30) Huang, L.; Allen, E.; Tonelli, A. E. Polvmer 1999, 40, 3211.

(31) (a) Okumura, H.; Kawaguchi, Y.; Harada, A. Macromolecules 2001, 34, 6338. (b) Porbeni, F. E.; Edeki, E. M.; Dan Shin, I.; Tonelli, A. E. Polvmer 2004, 42, 6907.

(32) Okumura, H.; Kawaguchi, Y.; Harada, A. Macromolecules 2003, 36, 6422

(33) Constable, G. S.; Lesser, A. J.; Coughlin, E. B. Macromolecules 2004, 37, 1276.

(34) $\overline{\mathrm{Xu}, \mathrm{H}}$.; Kuo, S. W.; Lee, J. L.; Chang, F. C. Macromolecules 2002, 35, 8788 . 
(35) Leu, C. M.; Chang, Y. T.; Wei, K. H. Chem. Mater. 2003, 15, 3721.

(36) Fu, B. X.; Lee, A.; Haddad, T. S. Macromolecules 2004, 37, 5211.

(37) Maitra, P.; Wunder, S. L. Chem. Mater. 2002, 14, 4494.

(38) Zhang, C.; Laine, R. M. J. Am. Chem. Soc. 2000, 122, 6979.

(39) Li, G.; Wang, L.; Ni, H.; Pittman, C. U., Jr. J. Inorg. Organomet. Polvm. 2001, 11, 123

(40) Nouvel, C.; Ydens, I.; Degee, P.; Dubois, P.; Dellacherie, E.; Six, J. L. Macromol. Symp. 2001, 175, 33.

(41) Kawaguchi, Y.; Nishiyama, T.; Okada, M.; Kamachi, M.; Harada, A. Macromolecules 2000, 33, 4472.

(42) Yun, X.; Painter, P. C.; Coleman, M. M. Polymer 1993, 34, 3010.

(43) Greene. T. W. Protective Groups in Organic Synthesis; John Wiley \& Sons: New York, 1981.

(44) Shin, I. D.; Huang, L.; Tonelli, A. E. Macromol. Svmp. 1999, $138,21$.

(45) Rusa, C. C.; Luca, C.; Tonelli, A. E. Macromolecules 2001 34,1318
(46) Hamadan, A. A.; Bugnon, P.; Sandan, C.; Lye, G. P.; Merbach, A. E. J. Am. Chem. Soc. 2000, 122, 592

(47) Huh, K. M.; Ooya, T.; Sasaki, S.; Yui, N. Macromolecules 2001, 34, 2402

(48) Rusa, C.; Tonelli, A. E. Macromolecules 2000, 33, 5321.

(49) Harada, K. Chem. Lett. 1984, 641.

(50) Gidey, M. J.; Bociek, S. M. J. Am. Chem. Soc. 1988, 110, 3820.

(51) He, Y.; Inoue, Y. Polym. Int. 2000, 49, 623.

(52) Shuai, X.; Porbeni, F. E.; Wei, M.; Bullions, T.; Tonelli, A. E. Macromolecules 2002, 35, 2401.

(53) Shuai, X.; Porbeni, F. E.; Wei, M.; Bullions, T.; Tonelli, A. E. Macromolecules 2002, 35, 3126.

(54) Crescenzi, V.; Manzini, G.; Calzolari, G.; Borri, C. Eur. Polym. J. $\mathbf{1 9 7 2 , 8 , 4 4 9 .}$

(55) Li, J.; Chenb, B.; Wang, X.; Goh, S. H. Polvmer 2004, 45, 1777.

MA050036H 